\title{
Hadron Electro-production in Cold Nuclear Matter at HERMES
}

\author{
Yves Van Haarlem ${ }^{1}$ - on behalf of the HERMES collaboration \\ University of Ghent, Subatomic \& Radiation Physics - Proeftuinstraat 86, 9000 Gent, Belgium
}

\begin{abstract}
The influence of the nuclear medium on lepto-production of hadrons was studied in the HERMES experiment at DESY using semi-inclusive deep-inelastic scattering of $27.6 \mathrm{GeV}$ positrons off deuterium, helium, nitrogen, neon, krypton, and xenon targets. The differential multiplicity of krypton relative to that of deuterium has been measured for the first time for various identified hadrons $\left(\pi^{+}, \pi^{-}, \pi^{0}, \mathrm{~K}^{+}, \mathrm{K}^{-}, \mathrm{p}\right.$ and $\left.\overline{\mathrm{p}}\right)$ as a function of the virtual photon energy $v$, the fraction $\mathrm{z}$ of this energy transferred to the hadron, and the hadron transverse momentum squared $\mathrm{p}_{\mathrm{t}}^{2}$. By studying the attenuation of leading and sub-leading hadrons in various nuclei, it is investigated how the hadron absorption and energy loss mechanisms contribute to the formation of hadrons.
\end{abstract}

Keywords: Hadronization, nuclear attenuation, double hadron correlation

PACS: 13.87.Fh, 13.60.-r, 14.20.-c, 14.40.-n

\section{INTRODUCTION}

If hadronization occurs in a nuclear environment rather than in vacuum, additional processes may occur before the final-state hadron is formed. This can result in a modification of the quark fragmentation functions. Acquiring a good understanding of quark propagation in the nuclear medium is crucial for the interpretation of ultra-relativistic heavy ion collisions, and high energy proton-nucleus and lepton-nucleus interactions. Quark propagation in the nuclear environment involves processes like multiple interactions with the surrounding medium and induced gluon radiation, resulting in energy loss of the quark. If the (pre-)hadron is formed inside the nucleus, it can interact via the relevant cross section, causing a further reduction of the hadron yield. Therefore, quark and (pre-)hadron propagation in nuclei are expected to result in a modification of the leading-hadron spectra [1]. By studying the properties of the leading hadrons emerging from nuclei, information on the time-space picture of hadronization can be obtained.

The process of double hadron production in nuclei can provide information about how the energy loss and the absorption mechanism contribute to hadronization, e.g. if the two produced hadrons are correlated, the energy loss mechanism is expected to dominate over the absorption mechanism, because energy loss occurs before the prehadrons are formed.

Semi-inclusive deep-inelastic lepton-nucleus collisions are most suitable to obtain quantitative information on the hadronization process because no deconvolution of the parton distributions of the projectile and target particles is needed.

\footnotetext{
1 yvhaarle@mail.desy.de
} 


\section{DATA ANALYSIS}

The experimental results for semi-inclusive deep-inelastic scattering on nuclei are presented in terms of the hadron multiplicity ratio $\mathrm{R}_{\mathrm{M}}^{\mathrm{h}}$. This is the ratio of the number of hadrons of type $h$ produced per deep-inelastic scattering event on a nuclear target with mass number A to that from a deuterium (D) target. It depends on the leptonic variables $v$ and $\mathrm{Q}^{2}$, which are the energy in the target rest frame and the squared four-momentum of the virtual photon, respectively, and on the hadronic variables $\mathrm{z}=\mathrm{E}_{\mathrm{h}} / v$ and $\mathrm{p}_{\mathrm{t}}^{2}$, where $\mathrm{p}_{\mathrm{t}}$ is the hadron momentum component transverse to the virtual photon direction. The multiplicity ratio is defined as

$$
\mathrm{R}_{\mathrm{M}}^{\mathrm{h}}\left(\mathrm{z}, v, \mathrm{p}_{\mathrm{t}}^{2}, \mathrm{Q}^{2}\right)=\left.\frac{\mathrm{N}_{\mathrm{h}}\left(\mathrm{z}, v, \mathrm{p}_{\mathrm{t}}^{2}, \mathrm{Q}^{2}\right)}{\mathrm{N}_{\mathrm{e}}\left(v, \mathrm{Q}^{2}\right)}\right|_{\mathrm{A}} /\left.\frac{\mathrm{N}_{\mathrm{h}}\left(\mathrm{z}, v, \mathrm{p}_{\mathrm{t}}^{2}, \mathrm{Q}^{2}\right)}{\mathrm{N}_{\mathrm{e}}\left(v, \mathrm{Q}^{2}\right)}\right|_{\mathrm{D}},
$$

where $\mathrm{N}_{\mathrm{h}}$ is the yield of semi-inclusive hadrons in a given $\left(\mathrm{z}, v, \mathrm{p}_{\mathrm{t}}^{2}, \mathrm{Q}^{2}\right)$-bin, and $\mathrm{N}_{\mathrm{e}}$ the yield of inclusive deep-inelastic scattering leptons in the same $\left(v, \mathrm{Q}^{2}\right)$-bin.

The measurements described were performed with the HERMES spectrometer [2, 3, 4] using the $27.6 \mathrm{GeV}$ positron beam stored in the HERA ring at DESY. Contributions from target fragmentation were suppressed by requiring $\mathrm{z}>0.2$. The data have been corrected for radiative processes.

To study how the energy loss and absorption mechanisms contribute to hadronization, the double ratio for leading and sub-leading hadrons

$$
\mathrm{R}_{2 \mathrm{~h}}\left(\mathrm{z}_{2}\right)=\left(\frac{\mathrm{d}^{2} \mathrm{~N}\left(\mathrm{z}_{1}, \mathrm{z}_{2}\right)}{\mathrm{dN}\left(\mathrm{z}_{1}\right)}\right)_{\mathrm{A}} /\left(\frac{\mathrm{d}^{2} \mathrm{~N}\left(\mathrm{z}_{1}, \mathrm{z}_{2}\right)}{\mathrm{dN}\left(\mathrm{z}_{1}\right)}\right)_{\mathrm{D}}
$$

has been considered. The term $\mathrm{d}^{2} \mathrm{~N}\left(\mathrm{z}_{1}, \mathrm{z}_{2}\right)$ is the number of events with at least two hadrons ( $\left.z_{\text {leading }}=z_{1}>0.5\right)$. The term $\mathrm{dN}\left(\mathrm{z}_{1}\right)$ is the number of events with at least one hadron, with $\mathrm{z}_{1}>0.5[5]$.

\section{RESULTS}

For the first time the $v$-dependence of the multiplicity ratios were studied for identified neutral and charged pions, kaons, protons and antiprotons, as shown in Fig. 1-right. The corresponding z-dependence of $\mathrm{R}_{\mathrm{M}}^{\mathrm{h}}$ with $v>7 \mathrm{GeV}$ is shown in Fig. 1-left. In the bottom panels the average values for $\mathrm{Q}^{2}$ and $\mathrm{z}$ or $v$ are displayed for all data.

The results presented in Fig. 1 show that the multiplicity ratios for positive and negative pions are similar and consistent with the multiplicity ratio for neutral pions. However, $\mathrm{R}_{\mathrm{M}}^{\mathrm{h}}$ for positive kaons is significantly larger. An even larger difference is observed between protons and antiprotons. Also, a stronger attenuation was observed for targets with increasing atomic mass number A [8] (not shown).

A nuclear enhancement at high $p_{t}^{2}$ is observed in the HERMES data shown in Fig. 2left both for ${ }^{14} \mathrm{~N}$ and $\mathrm{Kr}$ nuclei. The data for $\mathrm{p}_{\mathrm{t}}^{2}<0.7 \mathrm{GeV}^{2}$ show the attenuation previously discussed, while the data for $\mathrm{p}_{\mathrm{t}}^{2} \geq 0.7 \mathrm{GeV}^{2}$ reflect the $\mathrm{p}_{\mathrm{t}}$-broadening attributed to multiple scattering effects. This broadening is also called the Cronin effect and has previously been observed in heavy-ion and hadron-nucleus induced reactions. In contrast 

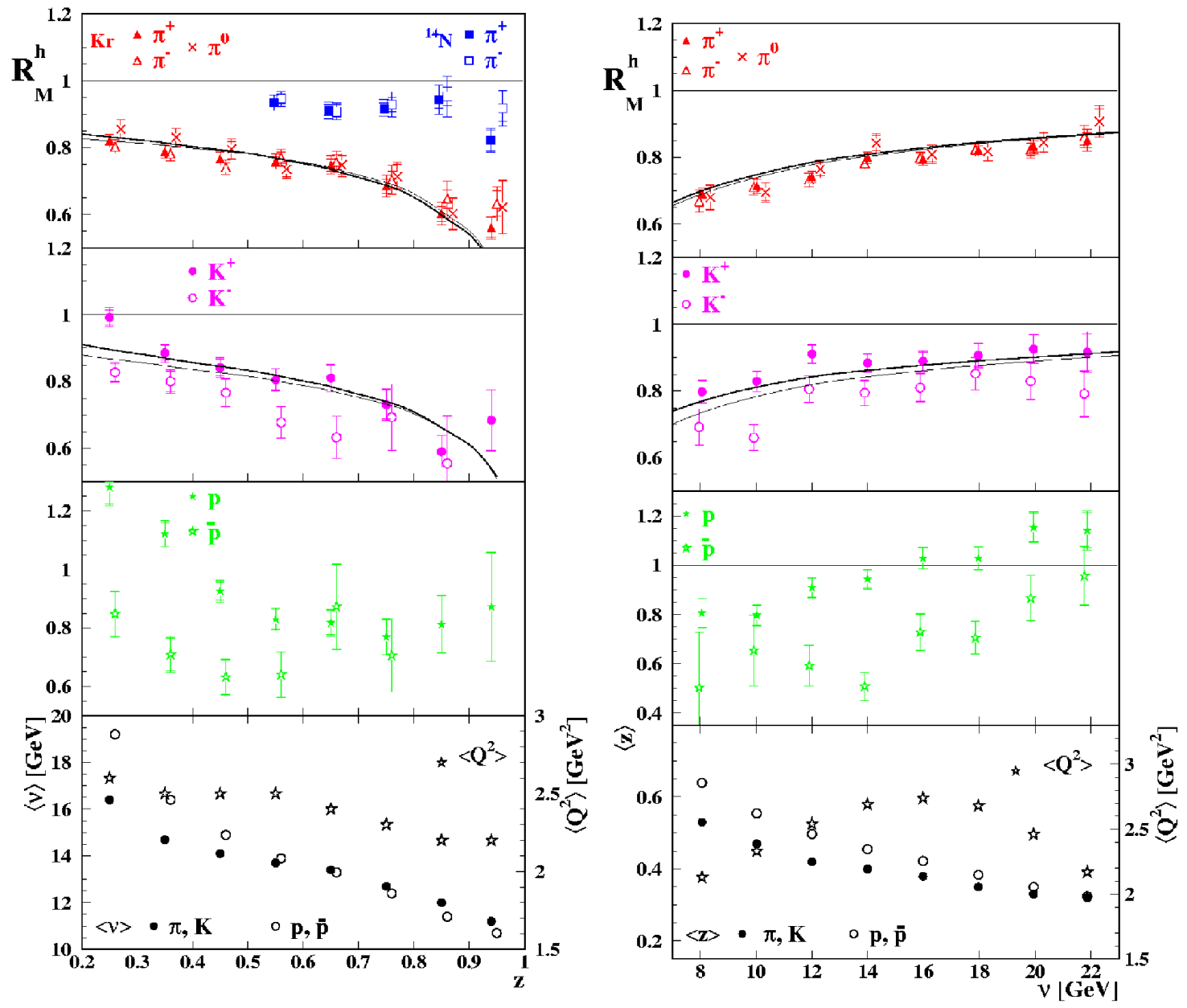

FIGURE 1. Multiplicity ratios for identified pions, kaons, protons and antiprotons from a $\mathrm{Kr}$ target as a function of $v$ for $\mathrm{z}>0.2$ (right), and as a function of $\mathrm{z}$ for $v>7 \mathrm{GeV}$ (left). In the upper panels the ratios for pions, kaons and protons are shown. In the bottom panels the average $\mathrm{z}$ and $v$ values are displayed for pions, kaons, and protons. The inner (outer) error bars represent the statistical (total) uncertainties. The thick (thin) solid curves represent the calculations of Ref. [6] for positive (negative) charge states.

to the previous reactions the Cronin effect at HERMES can only be due to final state interactions.

The results on $R_{2 h}\left(z_{2}\right)$, displayed in Fig. 2-right, show a reduction of $R_{2 h}$ compared to unity and essentially no A dependence [5].

\section{SUMMARY}

The multiplicities of identified hadrons on krypton relative to deuterium were measured for the first time. Different multiplicity ratios were observed for various hadrons.

The hadron multiplicity is observed to be enhanced at high $\mathrm{p}_{\mathrm{t}}^{2}$ in the nuclear medium, showing evidence for the Cronin effect in deep-inelastic scattering process, which can 

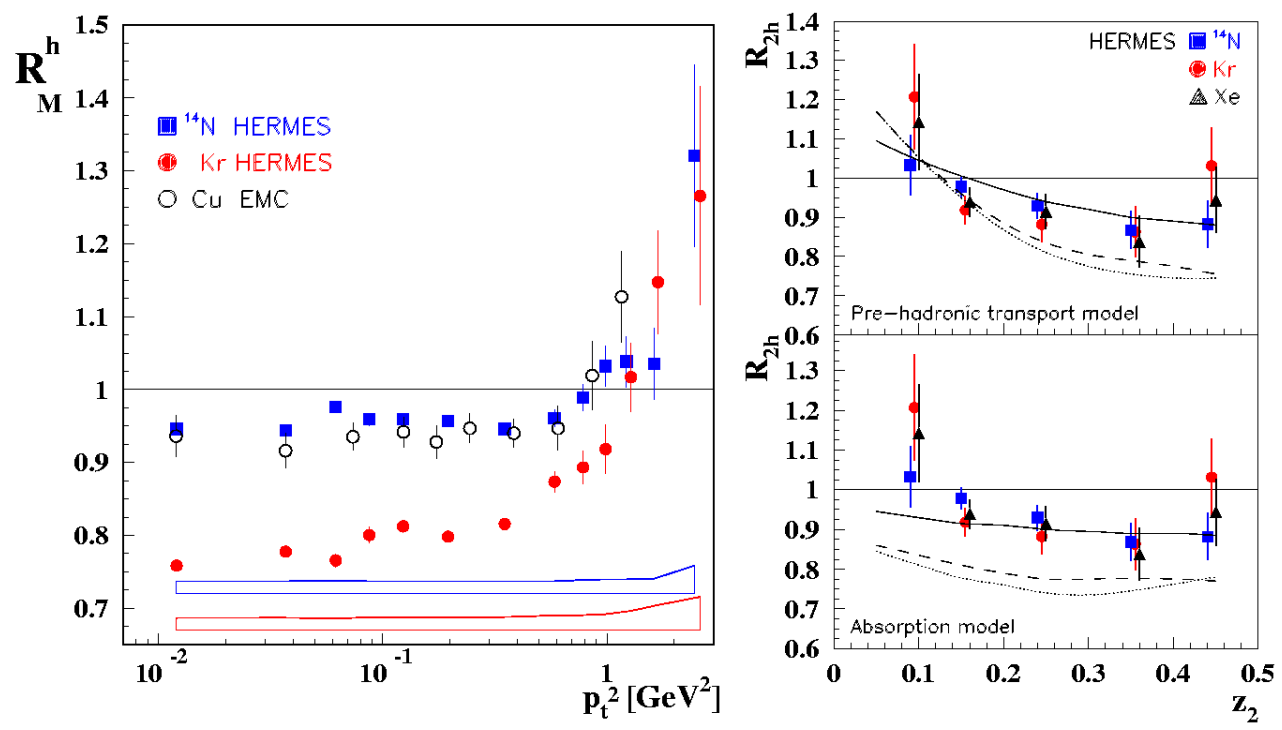

FIGURE 2. Left: Multiplicity ratio for charged hadrons versus $p_{t}^{2}$ for $v>7 \mathrm{GeV}$ and $\mathrm{z}>0.2$. The HERMES data on $\mathrm{Kr}$ and ${ }^{14} \mathrm{~N}$ are compared to the EMC data on $\mathrm{Cu}$. The error bars represent the statistical uncertainties. The systematic uncertainty for $\operatorname{Kr}\left({ }^{14} \mathrm{~N}\right)$ is shown as the lower (upper) band. Right: $\mathrm{R}_{2 \mathrm{~h}}$ as a function of $\mathrm{z}_{2}$ for ${ }^{14} \mathrm{~N}, \mathrm{Kr}$, and Xe. The curves (solid for ${ }^{14} \mathrm{~N}$, dashed for $\mathrm{Kr}$, dotted for Xe) are calculated within a BUU transport model [9] (upper panel), assuming only absorption [9] (lower panel).

only be due to final state interactions.

The ratio of leading and sub-leading hadron $R_{2 h}$ has been proposed, for the first time, as a tool to disentangling different mechanisms. The data show a reduction of $\mathrm{R}_{2 \mathrm{~h}}$ compared to unity and essentially no A dependence. These results, according to Ref. [10], suggest that a contribution from rescattering of the (pre-)hadron is present in the nuclei considered here.

\section{REFERENCES}

1. HERMES Coll., A. Airapetian et al., Eur.Phys.J. C21 (2001) 599-606.

2. HERMES Coll., K. Ackerstaff et al., Nucl. Instr. and Meth.A417 (1998) 230.

3. N. Akopov et al., Nucl. Instr. and Meth. A479 (2002) 511.

4. H. Avakian et al., Nucl. Instr. and Meth.A417 (1998) 69.

5. HERMES Coll., A. Airapetian et al, Phys. Rev. Lett. 96 (2006) 162301.

6. A. Accardi, V. Muccifora, H.J. Pirner, Nucl. Phys. A720 (2003) 131, and nucl-th/0211011.

7. X.N. Wang and X. Guo, Nucl. Phys. $\mathbf{6 6 9 6}$ (2001) 788; E. Wang and X.N. Wang, Phys. Rev. Lett. 89 (2002) 162301 and X.N. Wang private communication.

8. HERMES Coll, A. Airapetian et al, Phys. Lett. B577 (2003) 37-46

9. T. Falter et al., Phyus. Lett. B 594 (2004) 61; Phys. Rev. C 70 (2004) 054609; K. Gallmeister and W. Cassing, Nucl. Phys A 748 (2005) 241

10. B.Z.Kopeliovich, J.Nemchik, E.Predazzi, A.Hayashigaki, hep-ph/0311220 